\title{
La dialectique du modèle et de ses copies dans les Modellbücher du Berliner Ensemble
}

Un travail théâtral pionnier et son contexte

Die Dialektik von Modell und Nach-Bildung in den Modellbüchern des Berliner Ensembles: Situationen einer theatralischen Pionierarbeit

The dialectic of the model and its copies in Brecht's and the Berliner

Ensemble's Modellbücher: a pioneering theatrical work and its context

\section{André Combes}

\section{(2penEdition}

Journals

Édition électronique

URL : http://journals.openedition.org/ceg/415

DOI : $10.4000 /$ ceg. 415

ISSN : 2605-8359

\section{Éditeur}

Presses Universitaires de Provence

\section{Édition imprimée}

Date de publication : 5 avril 2017

Pagination : 149-170

ISBN : 979-10-320-0103-5

ISSN : 0751-4239

Référence électronique

André Combes, «La dialectique du modèle et de ses copies dans les Modellbücher du Berliner Ensemble », Cahiers d'Études Germaniques [En ligne], 72 | 2017, mis en ligne le 05 octobre 2018, consulté le 26 novembre 2020. URL : http://journals.openedition.org/ceg/415 ; DOI : https://doi.org/ $10.4000 /$ ceg. 415 


\section{La dialectique du modèle et de ses copies dans les Modellbücher du Berliner Ensemble}

Un travail théâtral pionnier et son contexte ${ }^{1}$

André COMBES

Université Toulouse Jean-Jaurès

Die Veränderungen des Modells, die nur erfolgen sollte, um die Abbildung der Wirklichkeit zum Zweck der Einflußnahme auf die Wirklichkeit genauer, differenzierter, artistisch phantasievoller und reizvoller zu machen, werden um so eindrucksvoller sein, da sie eine Negation von Vorhandenem darstellen - dies für Kenner der Dialektik

(GBFA 25, 389).

La recherche brechtienne s'est assez peu préoccupée d'interroger tout ce qu'implique, sur le plan théorique autant que pratique, la confection de Modellbücher ${ }^{2}$ dans la production et la transmission/réception du travail théâtral

1. Sur le travail de mise en scène de Brecht et du Berliner Ensemble dans les années 1949-1956 et les premiers Modellbücher, l'ouvrage de référence demeure Theaterarbeit, éd. par Ruth Berlau, Bertolt Brecht, Claus Hubalek, Peter Palitzsch et Käthe Rülicke en 1952. J'ai utilisé la troisième édition, revue et augmentée (Berlin, Henschelverlag Kunst und Gesellschaft, 1967), citée: TA $+\mathrm{n}^{0}$ de page. Par ailleurs, j'ai utilisé essentiellement les volumes 23 (Schriften 3 1942/431956), 25 (Theatermodelle et Katzgrabennotate), 27 (Journale 2, 1941-1955) et 30 (Briefe 3, 1950-1956) de la Große kommentierte Berliner und Frankfurter Ausgabe, éd. par Werner Hecht, Jan Knopf, Werner Mittenzwei, Klaus-Detlef Müller, Berlin / Weimar, Aufbau-Verlag, Frankfurt am Main, Suhrkamp Verlag, vol. 1-30, 1988-2000. Pour ne pas surcharger l'appareil de notes, j'indiquerai entre parenthèses: $G B F A+\mathrm{n}^{\circ}$ de vol. $+\mathrm{n}^{\circ}$ de page. Quand ils sont repris dans le volume 25 de la GBFA, je citerai parfois dans cette édition les textes de Theaterarbeit, ouvrage malheureusement épuisé.

2. Depuis le Modèle-Antigone (Antigonemodell) de 1948, réécriture de Sophocle dans la traduction de Hölderlin, les principaux Modellbücher ont été été rassemblés du vivant de Brecht dans les premières éditions de Theaterarbeit. Parmi les pièces de Brecht les plus importantes qui donnèrent lieu à la confection d'un Modellbuch, il faut signaler: Die Dreigroschenoper, Leben des Galilei, Mutter Courage und ihre Kinder, Der kaukasische Kreidekreis et Herr Puntila und sein Knecht Matti. Bien qu'en très grande partie numérisés, les Modellbücher existants ne sont, pour l'instant (automne 2016), consultables qu'au Brecht-Archiv (BBA) (Akademie der Künste). On peut y visionner un film récent sur le sujet: Peter Voigt / Sebastien Eschenbach, Bertolt Brecht. Bild und Modell (2006) (BBA - AVM13.0037). 
de Brecht entre son retour d'exil (1948) et sa mort (1956). Ces Modellbücher, qui avaient vocation à figurer la trace fouillée et systématique de ce travail théâtral sur chaque pièce mise en scène par le Berliner Ensemble, créé fin 1949 - qu'elle fût de Brecht ou d'un autre auteur -, ont pu donner lieu à la fabrication de Modèles ${ }^{3}$ rétrospectifs, chaque pièce jouée ou rejouée par la troupe étant par définition l'objet d'un nouveau travail modélisable. Ce fut ainsi le cas de $L a$ Mère de 1932, reprise en janvier 1951. De même, toutes les "remarques " sur les pièces et mises en scène des années cinquante sont implicitement des éléments constitutifs de Modellbücher, surtout quand elles sont accompagnées de photos de scène. La priorité donnée à la description détaillée et déjà modélisée des six premières mises en scènes du Berliner Ensemble dans le Theaterarbeit, publié en 1952, ne devait qu'en retarder la publication individuelle 4 .

Ces Modèles se concevaient assez ouvertement comme un modèle de travail théâtral et devaient manifester à quel point celui-ci était soucieux d'avoir la " maitrise de ses signes " (Roland Barthes) afin de générer un savoir social et politique sur le réel représenté. C'est pourquoi Brecht ne cessait de répéter qu'une première imitation active, celle dont la "fidélité " importe le plus, c'est celle du réel social qu'elle doit moins refléter que constituer en objet symbolique de pratique sociale transformatrice. C'est en ce sens que R. Barthes pouvait dire de la "dramaturgie brechtienne " en 1956 que "l'art dramatique a moins à exprimer le réel qu'à le signifier ". Dans les termes du $\$ 30$ du Petit Organon pour le théâtre (GBFA 23,77): cette dramaturgie devait être en mesure 1) de rendre ce réel "lisible " (sichtig) et non seulement "visible " (sichtbar) afin 2) de ménager la possibilité d'une "pensée intervenante " qui témoigne de l'engagement du théâtre dans ce même réel sous peine de ne pas pouvoir en donner des "représentations efficaces" (wirkungsvolle Abbilder) (ibid., § 23). Cela implique que sa reproduction scénique soit un travail spécifique sur la mise en signes qui ne se limite pas à une mimesis iconique visant à de purs effets de réel. Pas de simple découpe analogique de type naturaliste, de monstration d'une " tranche de réalité " produisant une reconnaissance plus qu'une connaissance du représenté. Pour être perceptible dans les Modèles des pièces, l'imitation inaugurale doit agencer dialectiquement l'espace scénique en segments sociaux et politiques signifiants. Elle le fait en s'adossant idéologiquement à la réception brechtienne du matérialisme historique et dialectique et, dramaturgiquement, en articulant ensemble une "fable " définie comme "Gesamtkomposition aller gestischen Vorgänge " (ibid.) et un "gestus fondamental " qui donne un sens social à ce que celle-ci " raconte" (voir infra).

Fut alors proposé à une certaine forme d'imitation apparemment directive un ensemble dont les principales composantes étaient d'abord le texte de la pièce accompagné d'un commentaire détaillé, jouant en partie le rôle de paratexte,

\footnotetext{
3. Pour traduire "Modell " dans le sens de "Modellbuch", j'écrirai " Modèle " avec une majuscule.

4. Cf. GBFA 25, 519.
} 
de didascalies ${ }^{5}$ plus interprétatives que descriptives. S'y ajoutaient parfois la description précise de la scénographie, comme pour celle de Caspar Neher dans le Modèle-Antigone (GBFA 25, 77-78), et surtout un grand nombre de photos de représentations ou de répétitions, séquences photographiques réalisées par Ruth Berlau, collaboratrice aussi remarquable qu'infatigable de ces Modellbücher ${ }^{6}$, puis par le décorateur-photographe Hainer Hill. On y trouvait encore, comme dans Theaterarbeit, des citations commentées de critiques ou de comptes-rendus comparatifs d'autres mises en scène avec, parfois, le dialogue instauré entre le Berliner Ensemble et des théâtres ouest-allemands ou étrangers sur l'utilisation des Modèles.

Les commentaires n'étaient pas destinés à expliquer les photographies mais à " relater et commenter " avec elles la représentation, la partie textuelle rappelant les principes qui ont orienté la conception de la mise en scène ${ }^{7}$.

Sans minimiser la fonction essentielle des photos de scène, il faut insister sur le rôle tout aussi capital du texte dans les Modellbücher. C'est lui qui fournit l'essentiel des significations de la représentation mais les photos n'en rappellent pas moins que le travail théâtral et un travail essentiellement visuel (plus qu'une visualisation) et que toute image scénique (même fixe et muette) produit du sens qui est en excès sur le texte, celui de la pièce comme celui des commentaires: elle peut en faire saillir des significations non verbalisées, mettre en critique le dialogue par des mimiques, des gestes ou un "arrangement scénique " particuliers. Ami de Brecht, le germaniste est-allemand Paul Rilla avait, dès 1950, explicité l'alliance du texte et de l'image à l'œuvre dans les Modèles:

5. Cf. la lettre de Brecht à Jan van Loewen du 4 octobre 1950: " My plays have sometimes failed even when staged by the best producers. One reason for this is that my scripts have very few stage directions. " (GBFA 30, 39). Comme le montre déjà le Modèle-Antigone, les nombreuses indications scéniques commentées des Modellbücher pallient largement l'absence quasi totale de didascalies de certaines pièces.

6. Le premier véritable Modellbuch, celui de l'Antigonemodell de 1948, paraît en 1955 au Henschelverlag de Berlin-est dans la série: "Ruth Berlau: Modellbücher des Berliner Ensemble ", publiée par la Deutsche Akademie der Künste. Les lettres de Brecht à R. Berlau des années 1950, si elles font état de leurs difficultés relationnelles croissantes, sont unanimes à reconnaître l'importance du travail de cette dernière - reconnue "inventeur du Modellbuch " (GBFA 30,417) - pour ce qui est de la confection des Modellbücher et de leur diffusion: "Entscheidend geholfen, die Idee von Modellaufführungen durchzusetzen. Entscheidend beteiligt an der Idee. Beteiligt bei ihrer Propagierung. Praktisch von allen Aufführungen [...]. Fotos gemacht, dazu Assistenten angelernt usw. Herausgegeben ein entscheidendes Buch 'Antigonemodell'. Vorbereitet ein zweites Buch 'Couragemodell'. Selber erste erfolgreiche Modellberatung in Westdeutschland durchgeführt. Selber beste Modellaufführung in Ostzone inszeniert ('Mutter'). " (Lettre du 22.2.1950, in GBFA 30, 14).

7. Sur le rapport des photos de scène et des textes des Modellbücher, voir la lettre de Brecht à Peter Suhrkamp de février 1950 sur le Modèle-Courage: "Es werden nicht Erklärungen der Bilder sein, sondern Text wie Bilder sollen getrennt die Aufführungen reportieren und kommentieren. Der Text mehr das Prinzipielle der Regie geben... " (GBFA 30, 16). Dans ce même Modèle, en ce sens lui-même exemplaire, Brecht décrit au début de chaque scène, qui reprend le texte et les didascalies initiales, un " arrangement de base " (Grundarrangement) pour indiquer comment le gestus fondamental de la scène doit être concrétisé scéniquement. 
Man verfolgt im Bild das Arrangement der Aufführung, die räumliche Gliederung, die Handhabung des Requisits, den Gestus der Solisten, die Führung der Gruppen, - und man verfolgt im erklärenden Text, was damit bezweckt ist, wohin die Aktion der Fabel zieht, aus welcher Handlung des Schauspielers das gesprochene Wort zu seiner Bühnenbedeutung, das heißt zu seiner Wirklichkeitsfunktion kommt, die eine gesellschaftliche Aussage ist. ${ }^{8}$

Avant d'être proposé à une réception imitative de cet ensemble, le Modèle concerne donc au premier chef son élaboration. Si on peut considérer que la confection de tels Modèles relevait d'un projet théâtral totalisant, on peut aussi y voir un modèle de production de signes et de significations mûrement pensée et discutée collectivement. La conscience de l'importance qualitative et quantitative de ce travail, qui savait et pouvait se donner le temps nécessaire à son accomplissement, a fondé Brecht et son équipe à demander aux mises en scène extérieures de le prendre en considération dans leur propre travail théâtral. Grand thuriféraire d'un "standard technique " de l'art en prise sur les progrès idéels et matériels des sociétés, Brecht voulut signifier dans ses Modèles sa volonté de ne pas revenir en deçà de ce qu'ils considérait comme des acquis théâtraux pleinement contemporains de leur temps: "sie (seine Modelle) waren Dokumente einer geleisteten Arbeit, hinter die nicht mehr zurückzufallen waren. ", écrit justement Jan Knopf 9 .

Soucieux d'instaurer autour des mises en scène de ses pièces une sorte d'interscénarité à valeur interdiscursive avec le Modèle, Brecht pouvait même préférer, à l'occasion, les tester sur des scènes extérieures ${ }^{10}$ avant de les inscrire au programme du Berliner Ensemble. Nous verrons qu'il s'agissait moins d'inciter à imiter un produit fini, appelé Modellbuch, qu'à procéder à une imitation indirecte: si le Modellbuch modélise un travail théâtral qui se veut une variante parmi d'autres, c'est celui-ci en tant que tel qu'il faut imiter et pas son résultat.

Wir entliehen diese Foto-Modellbücher an Theater, die nach uns das Stück inszenieren wollten. So haben sie eine doppelte Bedeutung: Einmal dokumentieren sie die Arbeit des Berliner Ensembles, und zum andern können sie eine Unterstützung und Anregung für Regisseure sein, die sich am gleichen Objekt versuchen. Leider sind diese Modellbücher oft in dem Sinn mißverstanden worden, als habe Brecht gewollt, da $\beta$ man ihn nachahmt; Er verstand sie aber als ein Angebot, auf seiner Arbeit aufzubauen.[...] Er wollte lieber vorher irgendwo Erfahrungen sammeln, also ein veränderbares Modell schaffen. ${ }^{11}$

Dans cette logique, Brecht avait dit par exemple, dès l'avant-propos du ModèleAntigone, que le jeu d'Helene Weigel dans les mises en scène de Die Mutter et de Die Gewehre der Frau Carrar à Copenhague par R. Berlau avait " créé quelque chose à la fois d'imitable et de modifiable " (etwas veränderbar Nachahmbares) (GBFA 25, 77). L'imitable comme transformable par des ajustements pertinents,

8. Paul Rilla, "Bühnenstück und Bühnenmodell ", Berliner Zeitung (DDR) du 7 mars 1950, p. 343 (BBA 1135/231 - 1135/237).

9. Jan Knopf, Brecht-Handbuch. Theater, Stuttgart/Weimar, Metzler Verlag, p. 2.

10. "Es waren Versuche, Modellaufführungen des Berliner Ensembles auf andere Bühnen zu übersetzen, und Versuche, Stücke an anderen Bühnen auszuprobieren, um Erfahrungen, Modellwerte für die betreffende Aufführung am Berliner Ensemble zu gewinnen. " (TA, 428).

11. Brechts Lai-Tu. Erinnerungen und Notate von Ruth Berlau, éd. avec une postface par Hans Bunge, Darmstadt und Neuwied, Luchterhand Verlag, 1985, p. 223 et 228-229. 
c'est la définition brechtienne de l'imitation d'un Modèle. En point de fuite de ses ajustements et réajustements internes ou externes, l'exergue le signifie avec emphase, il s'agira d'obtenir des représentations du réel " plus précises, plus différenciées, plus inventives et plaisantes sur le plan artistique ". Mais tous ces ajustements du Modèle, l'exergue y insiste également, n'ont de pertinence esthétique qu'en extériorité: ils doivent impérativement être surdéterminés dialectiquement par la négation du réel existant.

Dans ce contexte, on peut faire l'hypothèse que vouloir proposer à d'autres praticiens des modèles de travail théâtral est un indice culturel fort, ancré dans une situation historique où les dévastations idéologiques autant que guerrières semblaient devoir imposer une réfection généralisée de l'ensemble des secteurs de la société. Désorientations et réorientations pouvaient ainsi susciter un désir général de disposer de modèles fiables et pérennes dont le Berliner Ensemble a pu imaginer figurer l'embrayeur sectoriel : d'abord dans le périmètre restreint de la scène est-allemande, qu'il entendait contribuer à vivifier et régénérer, mais peut-être aussi au plan international où il opérait fréquemment (cf. TA, 328-362), avec un grand succès auprès de la critique comme du public: Paris, Londres, Stockholm, Moscou, Prague, Varsovie, Genève, Venise, Vienne, Budapest furent, entre autres, des lieux de présentation et de débats qui concernaient autant les pièces que le travail théâtral dont elles avaient été l'objet ${ }^{12}$.

Mais l'une des raisons principales pour lesquelles Brecht et ses collaborateurs entreprennent, au sortir de la Deuxième Guerre mondiale, de "modéliser " certaines représentations tient en grande partie aux conséquences idéologiques et politiques de la récente débâcle du nazisme. Brecht constate très vite qu'elle laisse l'ensemble de l'institution théâtrale - pièces, mises en scène et jeu de l'acteur - dans un état de délabrement artistique généralisé. N'avaient survécu qu'une grandiloquence creuse, aussi routinière qu'arbitraire, propre à alimenter une nouvelle fois un "théâtre culinaire " supposé satisfaire un public avide d'effets en tous genres hormis les effets de sens social. Le chapitre "Modelle " de Theaterarbeit souligne ainsi, avant toute chose, l'incapacité de théâtres dénués de " force, (de) sens artistique et (d') esprit progressiste " à mettre en scène les pièces anciennes ou nouvelles sans en proposer une "représentation fausse ":

\begin{abstract}
Außerstande, den Grundgestus eines Stückes [...], zu erfassen, kommen sie zu Aufführungen, deren Form nicht mehr die Form ihres Inhalts ist. Die szenischen Arrangements erzählen nicht mehr die Fabel, die Darstellung ist unrealistisch, die Details sind schlampig ausgeführt, ein unverbindlicher, künstlicher Schwung verhindert das Aufzeigen der Entwicklung der Figur oder einer Situation, die Aussage des Stückschreibers bleibt dunkel. ${ }^{13}$
\end{abstract}

Une autre raison essentielle voisine de proposer des Modèles était de battre en brèche les prestations souvent histrioniques d'acteurs prisonniers d'une tradition

12. Ce fut particulièrement le cas de la France lors des deux tournées parisiennes (1954 et 1960), couronnées par deux prix et admirées par les grands noms du théâtre populaire, Jean Vilar, Roger Planchon ou Jean-Marie Serreau, ainsi que par des grands critiques et théoriciens du théâtre comme Bernard Dort (Lectures de Brecht) ou Roland Barthes (Essais critiques).

13. Cf. " Falsche Darstellung neuer Stücke ", in TA, 285. 
dépravée de l'empathie aristotélicienne que la " théâtralité du fascisme ", intra ou extra muros, avait su renforcer, comme l'avait donné à voir Der aufhaltsame Aufstieg des Arturo Ui (1941), qui deviendra l'un des plus grands succès du Berliner Ensemble: expression surjouée d'affects déconnectés du gestus fondamental et de l'action de la pièce, identification décuplée par une tension superficielle entre scène et salle et partant impropre à fonder une nouvelle manière (épique) de jouer. Dans l'avant-propos du Modèle-Antigone 1948, publié pour la première fois en août 1949, Brecht avait déjà souligné que les dommages matériels subis par les théâtres pendant la guerre étaient nettement plus visibles que ceux subis par le jeu de l'acteur sous le nazisme: la soi-disant " brillante technique " des comédiens formés dans les "théâtres de Göring ", qui visait essentiellement à " voiler les causalités sociales ", ne pouvait a priori servir à leur dévoilement ${ }^{14}$. Ce mélange arbitraire et "inextricable de styles " rendait un travail de fond avec les acteurs indispensable afın que la "stylisation " nécessaire à leur jeu n'abolisse pas le " naturel " mais au contraire le revitalise (GBFA 25, 79-80). Dans une "Introduction " non publiée du Modèle-Courage, Brecht précisera:

In der ersten Leseprobe, selbst wenn sie noch gar nicht wissen, wie es weitergeht, holen die (die Schauspieler) alles Temperamentmäßige aus ihren Rollen und steigern sich von Anfang an in ein ,tolles‘ Tempo hinein. Sie reißen mit. Die Handlung ist ihnen nur ein Vehikel für die Darstellung seelischer Zustände, besonders der heftigen Art, allenfalls eine Gelegenheit, Spannung einzubauen (GBFA 25, 521).

La création du Berliner Ensemble en 1949, et surtout son installation dans ses propres murs au Theater am Schiffbauerdamm à partir de 1954, va permettre à Brecht de tenter de procéder à un redressement théâtral de grande ampleur, théorique et pratique, articulé sur ses propres pièces ou celles d'autres auteurs ${ }^{15}$. Elle va permettre également de promouvoir un travail sur le jeu de l'acteur axé sur la "gestique " dans le cadre d'un théâtre épique et dialectique, dont les photos des Modèles auront à fixer les figures sans les imposer. D'un même mouvement, Brecht voudra également accomplir un travail pédagogique régulier auprès du public ${ }^{16}$.

Mais la décision de confectionner systématiquement des Modellbücher des représentations, tout en laissant le soin à de nouvelles mises en scènes de les " augmenter et de les épurer ", semble avoir été également dictée par une autre urgence conjoncturelle plus complexe à circonvenir: le sentiment aigu que la décrépitude persistante des conceptions et pratiques artistiques provenait non seulement de la grande "braderie culturelle du capitalisme tardif " à l'Ouest,

14. Cf. Antigonemodell 1948, in GBFA 25, 73.

15. Le meilleur exemple de ce travail sur chaque détail du jeu des acteurs et de la mise en scène pour faire émerger toutes les significations du " gestus fondamental " d'une pièce d'un autre auteur fut accompli sur Katzgraben de Erwin Strittmatter et consigné dans les KatzgrabenNotate de 1953 qui ont également valeur de Modèle (GBFA 25, 401-490).

16. Cf. le texte non publié du Modèle-Antigone: " Das Buch soll aber, darüber hinaus, auch dazu dienen, dem breiteren Publikum eine bessere Kennerschaft des Theaters im allgemeinen zu verschaffen und seine Genußfähigkeit zusammen mit seiner Kritikfähigkeit zu vertiefen. » (GBFA 25, 507). 
mais aussi des insuffisances idéologiques du socialisme. Brecht a toujours eu conscience de la nécessité de mener un combat théâtral sur deux fronts :

Diese Modelle und die Neueinstudierungen, die sie erweitern und säubern [souligné par A.C.], sind so nötig, weil die Künste auf Grund des kulturellen Ausverkaufs des Spätkapitalismus und trotz der emphatischen Aufnahme der Künste durch die neue Klasse, zumindest zeitweise, von schnellem Verfall bedroht scheinen.[...] Aber die neue Klasse tritt die Herrschaft zwar mit neuen guten Impulsen, aber auch mit künstlich verbildetem Geschmack an. ${ }^{17}$

Le travail théâtral intensif du Berliner Ensemble était ainsi sous-tendu par la conviction que ce travail avait d'abord vocation à être largement diffusé dans les théâtres de RDA pour les inciter à améliorer qualitativement leur propre pratique, hypothéquée par un héritage négatif (cf. GBFA 30, 279) ${ }^{18}$. C'est l'une des raisons du souci permanent de Brecht de faire jouer sa troupe dans les principaux théâtres d'Allemagne de l'est pour inaugurer une forme de pédagogie théâtrale matérialiste en rupture avec les conceptions dominantes des époques passées, en continuité avec ses propres expériences weimariennes et en accord avec ce qu'il percevait comme les nécessités d'un travail idéologique de fond indispensable à une "construction du socialisme" autant culturelle que socio-politique. Tout au long des années cinquante, Brecht n'aura de cesse, sa correspondance et ses journaux en témoignent, de prôner une (ré)éducation artistique non seulement de la population de RDA mais aussi et surtout de ses acteurs culturels, y compris les principaux: ceux qui siègent dans les instances décisionnelles. Cet impetus pédagogique général explique certainement en grande partie la volonté de proposer des modèles de ce qu'il connaît le mieux en espérant que les questionnements et modifications systématiques des options retenues dans son travail théâtral puissent avoir une pertinence plus générale et concerner toute pratique socialiste de transformation sociale ${ }^{19}$.

Le chapitre " Modelle " de Theaterarbeit fera donc logiquement de la critique de l'existant une phase initiale incontournable de l'élaboration d'un Modèle en décrivant la " fausse représentation de pièces nouvelles ": par exemple, une Mère Courage figée dans la posture d'une pietà ou d'une Niobé, d'un Muttertier et d'une mater dolorosa, qui recouvre d'un pathos excessif ce que le gestus fondamental de la pièce avait impérativement signifié : " Die Händlerin ist nicht

17. Une rencontre avec des étudiants de la Arbeiter-und Bauern-Fakultät en juin 1951, pendant le travail sur le Modèle-Courage et la reprise de la mise en scène de la pièce, conforte Brecht dans sa critique de la situation intellectuelle de la jeune RDA et son constat de l'incapacité à faire émerger un art progressiste et une pensée artistique qui le fonde: "Das Denken bleibt verkümmert, wo Denkprodukte auswendig gelernt werden. [...] Auch künstlerische Werke werden nicht eigentlich studiert, das Künstlerische an ihnen wird links liegengelassen. » (GBFA 27, p. 322).

18. Pour une réception / continuation, inspirée par le $T A$ et les Modèles, du travail théâtral de Brecht dans la RDA des années 1970, voir: Regisseure der DDR inszenieren Brecht. Materialien und Fotos zu zwölf Aufführungen, Berlin-RDA, Verband der Theaterschaffenden, 1978.

19. Dans l'édition de 1966 de Theaterarbeit, on pouvait encore lire: "Noch immer gilt, was Brecht bei der Gründung des Ensembles als Programm formuliert hat. Das Berliner Ensemble versucht mit seiner Theaterarbeit zum Aufbau eines neuen Deutschlands und eines nationalen Theaters beizutragen. " $(T A, 428)$. 
unschuldig am Tod ihrer Tochter ", ainsi que le souligne le commentaire d'une photo de mise en scène extérieure (TA 286). Il s'agit ici de montrer concrètement, photos à l'appui, la manière dont " des fausses représentations brouillent le sens d'une pièce ". Commentant deux séquences comparées de photos de la même scène - au Berliner Ensemble puis à Munich et dans deux théâtres de RDA (TA, 289-290) - Theaterarbeit illustre ainsi comment des différences de mimique et de gestuelle peuvent inverser le sens de cette scène.

Là contre, la modélisation doit permettre au théâtre brechtien de refuser que des mises en scène arbitraires et non maîtrisées en faussent les intentions signifiantes, résultats de longues réflexions théoriques dont L'Achat du cuivre (1938-1955) a pu figurer le chantier-laboratoire et le Petit Organon pour le théâtre (1948), contemporain du premier Modellbuch (Antigonemodell), la "substantifique moelle ". Résultats aussi d'un travail d'écriture particulièrement exigeant associé à une confection collective tout aussi exigeante du moindre signe scénique qui fait fond sur une lecture et une mise en débat des intentions du texte afın de veiller en particulier à ce qu'aucun effet esthétisant n'hypothèque l'appréhension du réel théâtralisé ${ }^{20}$.

À partir de la description du travail concret avec les comédiens, base incontournable du travail théâtral ${ }^{21}$, un texte fondamental de Theaterarbeit (GBFA 23, 162-166), "La mise en scène de Bertolt Brecht ", peut ainsi se lire sans peine comme une invitation implicite à prendre en modèle une telle conception de la mise en scène pour tous les gens de théâtre désireux de participer à une refondation matérialiste de la pratique théâtrale en RDA.

Que pouvait-on dès lors transmettre d'un travail théâtral collectif, aussi minutieux et méthodique qu'ouvert à l'évolutif, qui produisît une imitation non répétitive d'un " modèle " tout en permettant de cerner au plus près les implications scéniques de notions dramaturgiques que Brecht jugeait essentielles : la fable, le gestus fondamental, l'épique et le "gestique "? Dans cette "postconstruction " - " das Modell nachbauen ", dit Brecht (GBFA 35, 507) - ce sont, avons-nous dit, moins les produits que les modalités complexes de ce travail qui sont proposés à l'imitation sur d'autres scènes, est-allemandes, ouestallemandes ou internationales. Brecht leur proposera volontiers la présence de ses collaborateurs (R. Berlau, Benno Besson, Peter Palitzsch en Europe ou Eric Bentley aux États-Unis), comme conseillers en dramaturgie brechtienne ou

20. "So sind die sinngemäßen Gruppierungen auf unserm Theater nicht 'rein ästhetische' Erscheinungen, Effekte, formale Schönheit gebend. Sie gehören zu einem Theater der großen Gegenstände für die neue Gesellschaft..." (GBFA 23, 173).

21. Theaterarbeit dressera la liste très concrète de " tendances générales que le comédien devrait combattre ", ce que les photos de scène illustreront: " Nach der Bühnenmitte zu streben / Sich von Gruppen zu lösen, um allein zu stehen / Sich der Person zu nähern, zu der gesprochen wird /[...] Immer parallel zur Rampe zu stehen / Beim Schnellwerden laut werden / [...] Widersprüchliche Charakterzüge zu verwischen / Die Absichten des Stückschreibers nicht zu erforschen..." $(T A, 387)$. 
superviseurs de mise en scène ${ }^{22}$. Tout comme il pourra inciter d'autres théâtres à jouer ses propres pièces devant un public berlinois - Der gute Mensch von Sezuan par les Münchner Kammerspiele par exemple - considérant qu'il peut être instructif pour sa troupe et ses acteurs d'examiner de près un travail théâtral différent sur un même objet afın de pouvoir mesurer la " qualification (Eignung) théâtrale générale " du Berliner Ensemble (GBFA 30,364) et donc sa prétention à fonctionner comme un modèle.

Le Modèle brechtien ne fut donc qu'en partie un garde-fou censé empêcher les autres mises en scène de ses pièces de retomber dans les ornières de l'habitus théâtral dominant après la traversée du nazisme: incapacité à dégager le gestus fondamental d'une pièce, à composer des arrangements signifiants de la fable, à ne pas savoir véritablement construire scéniquement un personnage ou à le laisser déconstruire par les débordements arbitraires d'acteurs plus préoccupés d'effets de scène que d'effets de sens. Brecht insiste tout autant sur le fait que les Modèles n'ont pas pour objectif de bloquer les mises en scène futures dans une posture de simple exécution d'une partition aux notations contraignantes, censée brider tout ce qui pourrait relever de l'interprétation: il ne faut y voir qu'une " incitation " (GBFA 25, 520).

La " copie " d'un Modèle, qui fournit d'abord une armature dramaturgique et scénique pourvoyeuse d'effets de sens sociaux et politiques, n'a donc pas à se vouloir reproduction servile d'un système complexe de signes scéniques. Elle doit faire de l'imitation un schème re-productif - " Nachahmung " et " Nachbildung ", dit Brecht -, qui rende manifeste qu'une opposition dichotomique traditionnelle entre modèle et copie est peu dialectique si la copie, qui, en fin de compte, révèle le modèle comme tel, n'a pas elle aussi vocation à devenir modèle. C'est un aspect fondamental de cette dialectique qui ne privilégie cependant pas, comme la mimesis d'Aristote, la copie (immanente et pragmatique) par rapport à un modèle (idéal et transcendant) qui s'y abolirait.

Il faut ainsi débarrasser la notion brechtienne de Modèle de toute prétention à être un original dont toute copie, définitivement seconde et présumée secondaire par rapport au statut pré-existentiel du modèle ne pourrait être que "pâle " ou de l'ordre du simulacre. Il n'y a pas dans le Modèle d'excès de clarté ou

22. Une lettre de mars 1955 témoigne de la volonté brechtienne de recueillir un maximum d'informations sur les scènes étrangères désireuses de jouer ses pièces avant de donner son accord: "Bei jeder Verhandlung muß ich über den Regisseur, die Hauptdarsteller, den Ruf des Theaters und, [...], über den Übersetzer informiert werden. " (GBFA 30, 603). Par ailleurs, il demandera dès 1950 à R. Berlau de lui faire parvenir des photographies du travail théâtral de théâtres intéressés par ses pièces (cf. GBFA 30, 21).

Brecht enverra la même R. Berlau - déjà chargée d'une mise en scène de Mère Courage à Rotterdam fin 1950 avec pour mission d'éliminer tout ce qui, dans la traduction néerlandaise (GBFA 30,497), pourrait dénaturer le sens de la pièce - superviser une autre représentation néerlandaise de la même pièce (voir infra) ou conseiller dramaturgiquement un projet danois sur Mère Courage à l'automne 1953 en fournissant des documents sur le travail théâtral du Berliner Ensemble (GBFA 30,186). De même, il proposera en octobre 1951 la collaboration de Benno Besson au TNP de Jean Vilar pour aider celui-ci à prendre la mesure des "difficultés que doit surmonter une mise en scène de la pièce. " (GBFA 30, 91). 
de coloration qui condamnerait à la pâleur toute imitation. Dans les termes brechtiens du § 54 du Petit Organon, la matrice théorique de l'élaboration des Modellbücher, la copie ne serait pas du registre de l'" Abklatsch ", mais bien davantage de l'" Abbild " ou de l'" Abbildung ", "imitation " qui crée des formes en en décalquant d'autres et qui est éminemment un " processus de pensée ".

La présence du terme de "Vorlage ", à côté de "Modell " et de "Muster ", est ainsi moins le symptôme d'hésitations conceptuelles que le signe d'une conceptualité moins contraignante qu'il n'y paraît au premier abord et qui fait de l'imitation de ces modèles-là bien plus qu'une affaire de zélé copiste: elle implique autant une invitation à respecter impérativement certains éléments structuraux de la pièce mise en scène par l'auteur et son collectif qu'elle pousse à ne pas hésiter à en proposer des modifications - de la scénographie au jeu de l'acteur - génératrices d'effets de sens nouveaux. Tout ceci, dans la ligne de l'expérimentation constante d'un auteur qui dès les années 1930, avait qualifié ses œuvres de "Versuche ".

Par ailleurs, Brecht semble également soucieux de faire rapidement litière des connotations négatives liées à cette pratique métathéâtrale nouvelle que fut la confection de Modellbücher: la fidélité servile des imitations nuirait à la " liberté créatrice ". Dès 1948, dans l'avant-propos du Modèle-Antigone, il avait remis en question l'individualité de toute création, surtout théâtrale, en soulignant à la fois son fondement collectif et sa qualité de processus dialectique qui impliquait de la considérer dans sa continuité évolutive: "Der Schöpfungsakt ist ein kollektiver Schöpfungsproze $\beta$ geworden, ein Kontinuum dialektischer Art, so da $\beta$ die isolierte ursprüngliche Erfindung an Bedeutung verloren hat. " (GBFA 25, 76).

En juillet 1949, il bat à nouveau en brèche les « objections contre l'utilisation de Modèles " en assumant cette entrave autoritaire délibérée à une "libre créativité " débridée, espérant qu'elle évitera maint détournement de sens :

Als die Benutzung von Modellen von Aufführungen vorgeschlagen wurde, erhob sich ein lautes Geschrei, dies sei Diktatur, 'freies Schöpfertum' solle verhindert werden. Der Stückeschreiber, um ein allzu freies Herumschöpfen mit seinen Stücken zu verhindern, griff tatsächlich zu sanfter Erpressung, indem er das Stück einer Zeitlang nur Bühnen zur Verfügung stellte, welche die Vorlage benutzten. (GBFA 25, 393). ${ }^{23}$

Et il ajoute, en pointant tout ce qu'il y a de pensée élaborée initiale dans un Modèle, que celle-ci est propre à provoquer des contradictions qui contribuent à faire également des imitations un processus théâtral de pensée:

23. La réponse des Éditions Suhrkamp à une demande des Städtische Bühnen Freiburg im Breisgau de mettre en scène Mutter Courage a pu refléter un moment plus directif des intentions brechtiennes: "Der Autor hat ganz bestimmte Vorstellungen von der Inszenierung seiner Werke und wünscht keine individuelle Interpretation seitens der Regisseure. [...] Es befindet sich eine besondere Regiepartitur in Vorbereitung. Solange diese nicht vorliegt, ist es der Wunsch von Herrn Brecht, dass Frau Weigel, die Darstellerin der 'Courage', möglichst zu Beginn der Aufführungen an einem Abend gastiert und dabei einen Begriff von den Absichten vermittelt. " (BBA - 1118/83). 
Man verliert erstaunlich denkbar wenig mit dem Verzicht auf die völlige Freiheit der "schöpferischen Gestaltung ". Irgendwo, mit irgend etwas mu $\beta$ man doch auf jeden Fall anfangen; warum sollte es nicht etwas schon einmal Durchdachtes sein? Seine Freiheit gewinnt man dann schon durch den Widerspruch, der sich allenthalben in einem meldet und regt $(G B F A 25,174)$.

Ce qui impliquait de faire d'un Modèle et de son imitation un processus qui ne soit pas du registre du "poncif " (Schablone) et graverait dans le marbre les signes scéniques d'une première mise en scène, mais veillerait à ce que les " actes créateurs " devenus "processus " soient en mesure de fixer le rythme des modifications subies par les Modèles: «... an die Stelle der sporadischen und anarchischen Schöpfungsakte sollen Schöpfungsprozesse mit schritt- oder sprunghaften Änderungen treten. " (GBFA 25, 77).

Il faut ainsi prendre au pied de la lettre, et non comme un propos rhétorique, une remarque du Theaterarbeit $(T A, 346)$ : " Jedes Modell ist von vornherein als unfertig zu betrachten; gerade dass seine Mängel nach Verbesserungen schreien, sollte das Theater einladen es zu benutzen. ". Le recours à un Modèle ne fige et ne pérennise aucun sens préétabli d'une réalisation scénique, il s'engouffre dans ce qu'elle a fait apparaître de manques. L'imitation des Modèles ne doit donc pas être seulement mimétique mais analytique et critique.

S’il n’y a pas de prévalence du modèle sur la copie ou survalorisation du " prototype ", c'est que le Modèle, au sens de "Vorlage " doit susciter des imitations dignes de lui en ce sens qu'elles ont à faire saillir le " sens de la fable " avec " pureté " et " élégance " (GBFA 25, 393), comme le signifiait notre exergue. Il doit diffuser cette " passion du produire " et ce plaisir fort d'imitations inventives dont parlait avec emphase tout le début du Petit Organon.

Dans ce même esprit, le texte de couverture du Antigonemodell 1948, publié en 1955 au Henschelverlag, précise par exemple, à l'adresse du comédien, l'importance de trouver par son jeu des solutions artistiques qui améliorent le Modèle :

Die erläuternden Spielanweisungen stellen keine sklavisch zu benutzenden Gebrauchsanweisungen dar. Es steht dem Schauspieler frei, andere Spiellösungen zu finden, die künstlerisch treffend sind und den Sinn der Gesamtkonzeption wiedergeben [souligné par A.C.] (GBFA 25, 507).

Loin d'entraver la créativité, le Modèle brechtien entend donc la simuler en l'incitant à tester un grand nombre de solutions artistiques afın de choisir celles qui conviennent le mieux pour explorer le vaste champ des possibles scéniques. C'est pourquoi Brecht a donné dans un premier temps à son texte paru dans le Theaterarbeit sur la manière dont le metteur en scène Erich Engel " utilise le Modèle ", un titre révélateur: "Kopie eines Meisters ". La copie exécutée par quelqu'un qui a l'envergure d'un " maître " donne à celle-ci un statut analogue au " modèle " et à son auteur une sorte d'égalité créatrice par rapport à l'auteur du modèle initial: "Das Kopieren ist eine Kunst für sich, eine der Künste, die der Meister beherrschen muß. Er muß es schon deshalb, weil er sonst selbst nicht Kopierbares herstellen kann [souligné par A.C.] " (GBFA 25, 191). 
On se souvient que, chez Platon, la copie, certes plus proche du paraître que de l'être véritable, peut néanmoins être une fabrication efficace et fondée sur un savoir faire (technè) axé sur celui, plus théorique et scientifique, de l'épistémè. De même, la copie du modèle brechtien implique une égalité de compétences: une compréhension fine dudit modèle pour montrer à quel point il continue à être fécond dans ses transformations. Brecht est ici assez proche de la distinction platonicienne de la Politeia entre l'artiste, dont la représentation imitative du réel ne dit rien sur la substance matérielle de celui-ci, et l'artisan-démiurge:

Ein richtiger Handwerker versteht aber nach Platon etwas mehr von seinem Gegenstand, er versteht nämlich, wie es auch im griechischen Wort demiourgós schon im Namen liegt, etwas vom ,Werk' (érgon) einer Sache [...] weshalb Platon den Kenner des Werks eines Gegenstands der Idee näher rückt als einen, der blo $\beta$ äußere Erscheinungsformen zur Kenntnis nehmen will. ${ }^{24}$

Ainsi, si la nouvelle mise en scène a quelque chose à imiter et à apprendre du modèle, c'est la nécessité de considérer tout travail théâtral comme un "processus de production" fait de multiples expérimentations de solutions scéniques concrètes. En ce sens, toute imitation transformante fondée sur la plus concrète des pratiques scéniques peut se voir reconnaître le statut de modèle et le transformateur d'élève devenir maître: « Die Abänderungen, richtig vorgenommen, haben selber modellhaften Charakter, der Lernende verwandelt sich in den Lehrer, das Modell ändert sich." (TA, 346). Pas de copie digne de ce nom donc si son auteur n'a pas la maîtrise de son art, l'art théâtral: car "pureté " et " élégance " doivent être la marque de celui qui est capable d'investir entièrement l'" arrangement " initial pour le retravailler sans a priori, en soumettre tous les aspects à une expérimentation pratique qui augmente artistiquement sa valeur heuristique :

Meist gibt er [Erich Engel, le metteur en scène] irgendwelche vorläufige Stellungen an; dann studiert er sie und beginnt, hineinzugehen, das heißt Verbesserungen vorzuschlagen, die den Sinn der Fabel reiner und eleganter herausbringen. Bei dieser induktiven Probenart kann eine Vorlage kaum in die Quere kommen, sie dient einfach als die vorläufige Annahme, die studiert und korrigiert wird. [...] Das bedeutet aber, daß er imstande sein mu $\beta$, die Vorlage erst einmal nachzubauen, in einer lockeren Weise, als etwas Vorläufiges, jedoch Bedeutsames. (GBFA 25, 391-392). ${ }^{25}$

Par ailleurs, un véritable " maître " se doit de connaître non seulement les personnages et le texte de pièces " souvent jouées " mais aussi leurs différentes mises en forme théâtrale (theatralische Ausformung) afin d'insuffler dans " tout nouveau modèle " des éléments d'un travail théâtral plus ancien. Si la productivité intrinsèque d'un Modèle se mesure à son aptitude à être "imité " et " varié ", il doit également suggérer ce qui, de lui, ne gagne rien à l'être: rien n'oblige par exemple à copier les foulards d'Helene Weigel dans Mère Courage ni l'expression muette de son cri de douleur (GBFA 25, 394). Dans tout Modèle, il y a à la fois de l'» exemplaire " (imitable) et du " sans exemple " (inimitable):

24. Arbogast Schmitt, "Mimesis bei Platon ", in Gertrud Koch, Martin Vöhler, Christiane Voss (dir.), Die Mimesis und ihre Künste, München, Wilhelm Fink, 2010, p. 239.

25. Souligné par A. C. 
Ein Modell steht und fällt mit seiner Nachahmbarkeit und Variabilität. Er kann nicht auf Tonfällen beruhen, deren Reiz durch besondere Stimmen, auf Gesten und Gängen, deren Schönheit durch besondere körperliche Eigenschaften der Schauspieler entsteht: derlei hat nicht Modellwert, es ist beispiellos und nicht beispielhaft. Die aktuale Leistung bei Benutzung eines Modells muss eine Mischung von Beispielhaften und Beispiellosem sein $(T A, 346)$.

Pas d'imitation improbable de l'inimitable donc, soit les "spécificités corporelles " les plus individuelles du jeu de l'acteur: sa voix, sa gestuelle et sa démarche propres. Car la performance de l'acteur en tant que corps scénique pétri d'individuel - que l'on pense, pour s'en tenir à ceux du Modèle-Courage, aux deux corps si différents d'Helene Weigel (Berlin, 1949) et de Therese Giehse (Munich, 1950) - instille toujours intrinsèquement du performatif unique et difficilement copiable dans le modèle. On a alors affaire à un Modèle à deux ou plusieurs entrées, fonctionnant sur la base de ce différentiel irréductible: Theaterarbeit intitule "Exemples de variantes " toute une série de photos comparatives des mises en scène de Mère Courage à Berlin et à Munich avec leur descriptif commenté du gestus fondamental d'une dizaine de scènes de la pièce (TA, 316-326). Car les photos de scène sont aussi faites pour manifester le rôle essentiel du jeu comparé de l'acteur dans le dispositif scénique modélisé. Dans un texte du même ouvrage, "Wie benutzt der Regisseur Brecht sein eigenes Modell ", Brecht écrit à propos du Modèle-Courage:

\footnotetext{
Er (Brecht) studierte die Modellbücher, um den Grund herauszufinden, warum er zu dieser Lösung, zu dieser Stellung oder dieser Geste gekommen war. [...]: Modell hin, Modell her, sie (die Schauspieler) konnten zeigen, was sie wollten; wenn es gut war, wurde es sofort aufgenommen. Sofort gab Brecht jede alte Lösung auf, wenn er eine neue von den Schauspielern geliefert bekam, die den Sinn der Sache, den Gestus, den Inhalt wiedergab und die Chronik weitererzählte. (GBFA 25, 394).
}

Ici, apparaît clairement que, si collectif fût-il, le Modèle est confectionné sous la direction générale de l'auteur de la pièce, celui qui a priori non seulement en connaît le mieux le gestus fondamental dans ses articulations dramaturgiques mais en est aussi le premier metteur en scène (Antigone et Mutter Courage) et a donc pu en tester scéniquement texte et paratexte dans ses plus infimes détails, ce pourquoi il est le premier bénéficiaire du Modèle qui peut alors fonctionner comme outil de travail interne du Berliner Ensemble: "Ein Arbeitsbuch, eine Kladde zum Handhaben bei der Regie " (GBFA 30, 370).

Es (das Modell) ermöglicht das Auffinden übertriebener Gesten, unwahrer Ausdrücke, falscher Haltungen, guter Ausdrücke und charakteristischer Gesten. So bekommt nicht nur das fertige Modellbuch einen Wert, den für die Benützer, sondern es hat auch einen Arbeitwert, einen Lehrwert schon für die Hersteller. ${ }^{26}$

C'est le rôle essentiel que jouent les photos de scène pour tester le Modèle en vérifiant le bien fondé d'"attitudes, de gestes, de déplacements ou de groupements " :

26. R. Berlau, in $T A, 296$. 
... sondern wir benutzen von fotografierten Haltungen, Gesten, Gängen und Gruppierungen, was wir brauchen können, um die Wahrheit auf die Bühne zu bringen: schlechte Haltungen ebenso wie gute Haltungen, die schlechten, um sie zu verändern, die guten, um sie nachahmenswert zu machen. ${ }^{27}$

Et c'est aussi leur rôle, toujours à usage interne, d'aider à découvrir ce qui peut parasiter le Modèle: "die hingeschlampte kleine Szene im Hintergrund, den lieblos gemachten, nichtssagenden Stuhl ${ }^{28}$.

L'auto-imitation du Modèle par ses créateurs peut parfois rendre manifeste à quel point certaines spécificités sociologiques peuvent influencer les options scéniques : en réutilisant le Modèle-La Mère à Leipzig en 1950, Brecht put constater que, contrairement aux ouvriers-acteurs de 1932, les acteurs professionnels avaient tendance à s'extraire des regroupements scéniques pour dire leurs répliques $(T A, 337)$. Une autre différence entre le même Modèle et sa reproduction concernait les variations signifiantes de la diction de H. Weigel entre 1932 et 1951: l'évolution historique imposait à la fois répétition et différence pour ne pas occulter dans le gestus d'une scène les traces de la Seconde Guerre mondiale: " Zum Beispiel zeigte die Weigel die Agitation gegen den Krieg (13. Szene) 1932 heiter und freundlich - damals stand Berlin noch - jetzt ungeduldig und hart. " (TA, 341). Un respect formaliste du Modèle de 1932 aurait aboli toute possibilité de laisser percevoir l'inscription de l'histoire dans les vies réelles de ceux qui le disaient ou l'écoutaient. Un simple effet de diction suffit à le manifester.

En ce sens, de tels Modèles ne connaissent réellement qu'une forme de contrainte: que les imitations respectent les procédures dramaturgiques et les significations multiples qu'un certain type de travail théâtral élabore à partir d'un certain type de texte dramatique et de sa structure de base, sa fable et son gestus fondamental, gestus global qui se décline dans les gestus particuliers des situations dramatiques, des personnages mais aussi de la scénographie et des accessoires. Il n'y a donc rien d'étonnant au fait que c'est précisément au moment où il commence de formaliser son travail théâtral que Brecht définit avec le plus de clarté ce concept aussi complexe qu'essentiel - mais aussi si difficile à modéliser - dans deux brefs textes dactylographiés de 1951-52, "Gestik " et "Über den Gestus »:

Darunter verstehen wir einen ganzen Komplex einzelner Gesten der verschiedensten Art, zusammen mit Äußerungen, welcher einem absonderbaren Vorgang unter Menschen zugrunde liegt und die Gesamthaltung aller an diesem Vorgang Beteiligten betrifft [...] oder einen Komplex von Gesten und Äußerungen, welcher, bei einem einzelnen Menschen auftretend, gewisse Vorgänge auslöst (die zögernde Haltung des Hamlet, das Bekennertum des Galilei usw.) oder auch nur eine Grundhaltung eines Menschen (wie Zufriedenheit oder Warten). Ein Gestus zeichnet die Beziehungen von Menschen zueinander. Eine Arbeitsverrichtung ist kein Gestus, wenn sie nicht eine gesellschaftliche Beziehung enthält wie Ausbeutung oder Kooperation. ${ }^{29}$

27. Brechts Lai-Tu, p. 283.

28. Ibid.

29. "Gestik ", in GBFA 23, 188. 
Tous les gestus ont une dimension socio-historique précise, incarnée performativement par ce qui organise le jeu corporel du comédien : attitudes, déplacements, gestuelle, mimique, diction, costumes et accessoires ${ }^{30}$. Il faut noter ici que l'importance de ces derniers ne doit pas être négligée car ils ont non seulement "à manifester à chaque occasion le gestus social de la pièce ${ }^{31}$ " mais aussi à montrer la manière dont les attitudes corporelles d'un comédien photographié dans plusieurs rôles entretiennent une relation étroite avec ces costumes et accessoires (cf. TA, 348-386). Theaterarbeit décrit comment R. Berlau a dû convaincre une comédienne hollandaise qui jouait Mère Courage que les accessoires ne sont pas une entrave au jeu du comédien lors des répétitions mais déterminent en partie celui-ci en matérialisant certaines significations de la fable ${ }^{32}$. La longue séquence de photos de $\mathrm{H}$. Weigel dans différentes scènes de la pièce, à laquelle font suite un texte sur la "langue de la Weigel " et le poème de L'Achat du cuivre sur "Die Requisiten der Weigel ", a, dans ce contexte, pleinement valeur de modèle ${ }^{33}$.

On peut estimer que la composition propre aux Modèles, et c'est certainement leur grand enseignement, privilégie la lisibilité spécifique des différents gestus par rapport à la fable, qui vise obstinément à unifier et homogénéiser les significations singulières de la pièce. Les Modellbücher œuvrent ainsi à interroger, remettre en question, réinterpréter et préciser par petites unités gestiques ce que la fable a toujours tendance à totaliser. C'est un travail de découpe et de vérification

30. Dans son entrée « Geste/Gestus », le Metzler Lexikon Theatertheorie (éd. par Erika Fischer-Lichte, Doris Kolesch, Matthias Warstat, Stuttgart/Weimar, Metzler, 2014, p. 140) affirme: "Auf der Bühne des epischen Theaters soll die Mimik als Spiegel der individuellen Seele von der Gestik als Zeichen sozialhistorisch typischen Verhaltens ersetzt werden ". Mais, dans le théâtre brechtien, mimique et gestuelle sont plutôt complémentaires : le meilleur exemple en est la séquence intitulée " Gestisches " du Modèle-Courage (GBFA 25, 342-350).

31. Selon les termes de l'article novateur de R. Barthes, "Les maladies du costume de théâtre ", paru dans la revue Théâtre populaire en 1955, après la tournée parisienne du Berliner Ensemble. Barthes fut l'un des tout premiers à comprendre l'importance des costumes pour le théâtre brechtien et à les poser implicitement en modèles à imiter dans leur matérialité signifiante: "...le costume de théâtre doit être assez matériel pour signifier et assez transparent pour ne pas constituer ces signes en parasites. ", in Roland Barthes, Essais critiques, Paris, Éd. du Seuil, 1964, p. 53 et 61.

Les deux descriptions des costumes de la Mère et du Puntila dans Theaterarbeit (TA 361, 363) donnent une idée précise du soin apporté au traitement chimique et mécanique des costumes pour leur conférer un gestus particulier et peuvent, en tant que procédé et non comme esthétique spécifique, servir de modèle. L'économie symbolique des costumes et accessoires de la scène brechtienne en propose ce que S. Trétiakov, l'ami de Brecht, appelait " biographie de l'objet ". Un des meilleurs exemples: le drapeau rouge de La Mère de 1932 apparaît en 1951, après la traversée du nazisme et de la Seconde Guerre mondiale, comme un emblème défraîchi. Dans sa mise en scène de la pièce en 1970, Peter Stein, nouveau contexte historicopolitique oblige, lui restituera l'éclat vif du neuf.

32. R. Berlau note avec satisfaction que l'accessoiriste hollandais a immédiatement compris l'enjeu, affirmant: " unsere Liste der Requisiten gebe schon allein die Fabel des Stückes her " (TA, 329).

33. De ces accessoires, les photos de scène du Couragemodell en montrent le " détail ", toujours en plan moyen d'un ou deux personnages. Une hyperfocalisation par un gros plan sur l'objet donnerait à voir plus pour signifier moins. 
permanente des agencements les plus concrets des matériaux scéniques que le chapitre "Modelle des Berliner Ensembles " de Theaterarbeit décrit en listant le séquençage des tâches et les objectifs qui président à la gestation du Modèle: il doit donc privilégier le particulier le plus concret, s'attacher au " traitement des détails ", proposer une "division précise du mouvement des actions " tout en " indiquant le tempo et le déroulement de la représentation" (TA, 296-302) ${ }^{34}$. Ce sera en très grande partie la fonction complexe des séquences de photos de scène.

De par leur systématicité et leur ampleur, ce sont celles-ci qui constituent la grande nouveauté des Modèles. Plutôt que de les appeler improprement " photogrammes ", car elles ne sont pas extraites d'un film, on pourrait plutôt les qualifier de "photos-modèles ${ }^{35}$ ".

$\mathrm{Si}$ on se souvient de la suspicion brechtienne envers le média photographique - "Die Fotografie ist die Möglichkeit einer Wiedergabe, die die Zusammenhänge wegschminkt " (GBFA 21, 443) - force est de constater que les photos des Modellbücher, adossées à leurs commentaires, rétabliront les " rapports " manquants. Mais même montées en séquences qui simulent le montage, comme celles de la scène 12 de Mutter Courage (GBFA 25, 295-301), elles demeurent des photos individuées. Cadrées la plupart du temps en plan moyen et en légère contreplongée, ces photos présentent peu d'effets de cadrage et peu de changements d'angle de prise de vue. Comme le voulaient Brecht et R. Berlau, elles adoptent une perspective fixe depuis un point de vision jugé idéal dans la salle, les éclairages mal réglés étant un problème récurrent de leur confection ${ }^{36}$.

Pas de gros plan ni de plan général donc, pour être au plus près du gestus de la scène photographiée tout en évitant l'empathie tendancielle du gros plan, pour délivrer donc plutôt de la " signifiance " (globale) que des " significations " (isolées) ${ }^{37}$. Il y a beaucoup de visages baissés dans ces plans moyens qui exhaussent la gestuelle plus que la mimique, la proxémique plus que les postures. Ces photos-modèles, qui obéissent au principe de l'instantané photographique,

34. Sans aller jusqu'à parler comme lui d'“ opposition irréductible ", je suivrais ici Hans-Thies Lehmann quand il écrit de la recherche brechtienne: "Sie hat kaum die Möglichkeit in Betracht ziehen wollen, dass zwischen dem, was Brechts Idee des Gestus anzielt, und seinem Konzept der Fabel nicht ein Verhältnis der harmonischen Konsequenz, vielleicht sogar ein unüberbrückbarer Gegensatz bestehen könnte." (H.T. Lehmann, Das politische Schreiben, Berlin, Theater der Zeit, 2002, p. 231). Mais c'est précisément dans l'alliance non " harmonieuse " des commentaires et des photos des Modellbücher que se manifeste à quel point le théâtre brechtien est à la fois un théâtre de la fable et un théâtre du gestus.

35. Cf. Roland Barthes, "Sept photos-modèles de Mère Courage " (sur des photographies de PIC), in Euvres complètes, Paris, Éd. du Seuil, 1993, t. I, p. 833/847.

36. R. Berlau se plaint souvent des mauvaises conditions d'éclairage (comme des défauts de son vieux Leica), en particulier pour les photos du Modèle-Antigone. Brecht le confirmera pour le Modèle-Courage, photographié avec l'éclairage insuffisant du Deutsches Theater: arrièreplan et décors circulaires assombris, impossibilité de rendre "la lumière mate et dorée " de l'original scénique (GBFA 25, 172).

37. Cf. Roland Barthes, "Le troisième sens. Notes de recherche sur quelques photogrammes de S. M. Eisenstein ", Cahiers du cinéma, n² 222, juillet 1970. 
ne rendent pas le mouvement mais peuvent le laisser deviner dans certains cas par la position corporelle, les déformations des vêtements voire certains effets de filé ${ }^{38}$. R. Berlau nomme ce procédé, pour elle éminemment réaliste: " photographier dans le mouvement":

Kann man ein Drama fotografisch festhalten. [...] Wenn man in die Bewegung hineinfotografiert, behaupte ich, ist es möglich, insbesondere, wenn es sich um epische Stücke, epische Regie und epische Darstellungskunst handelt. Wenn die Aufnahmen gestellt sind, entsteht ein haarscharfes, aber verfälschtes, unrealistisches Bild. ${ }^{39}$

Par ailleurs, on peut remarquer ici, dans le sillage de W. Benjamin, que la confection d'un Modèle étayé par la seule visualité photographique est en soi un procédé parfaitement épique: en interrompant le déroulement de l'action et en "gelant " les mouvements pour produire des " effets de tableau ", un tel Modèle permet de cadrer et fixer cette "gestique " qui est au cœur des effets de sens de la mise en scène - "Gesten erhalten wir um so mehr, je häufiger wir einen Handelnden unterbrechen ${ }^{40} "$ - induisant un réalisme paradoxal qui fait abstraction de ces deux composantes essentielles que sont la voix et le mouvement relégués dans les commentaires ${ }^{41}$.

Ceci présente néanmoins quelques avantages : "Auf den Bildern kann nicht das Wort oder der Schwung über die Dürftigkeit des Anblicks hinwegtäuschen ", écrit R. Berlau dans un texte fondamental de Theaterarbeit (GBFA 25, 532). Se pourrait-il que cette absence de la voix de l'acteur telle qu'en sa diction, jointe à l'absence d'un mouvement autre qu'esquissé, permette aux photos d'affûter la dialectique du visible et du lisible d'un théâtre où la verbalité n'est plus parasitée par la verbosité ni la gestuelle par la gesticulation? Les photos de scène assureraient ainsi cette disjonction du voir et du parler, du visuel et du sonore, chère à Deleuze, idée-cinéma par excellence mais que ces photos importent dans le théâtre.

Par ailleurs, il peut y avoir intérêt à proposer des visualités muettes et inanimées pour tester le "rythme de la narration " et la plasticité des scènes (TA, 234). Les photos de scène auront alors à donner une vision " chorégraphique " du gestus.

38. Par exemple le Créon du Modèle-Antigone (GBFA 25, 137,139 et 141). Les exemples les plus parlants se trouvent dans la séquence de photographies intitulée "Bewegte Vorgänge " du Modèle-Courage (GBFA 25, 353-355), peut-être le travail photographique le plus remarquable de R. Berlau.

39. Brechts Lai-Tu, p. 280-281.

40. Cf. Walter Benjamin, "Was ist das epische Theater?", in Gesammelte Schriften, éd. par R. Tiedemann et H. Schweppenhäuser, Frankfurt am Main, Suhrkamp, 1980, T. II. 2, p. 534-536 et p. 521 .

41. Cf. les textes de Theaterarbeit: "Aus einem Brief an einen Schauspieler ", "Kontrolle des 'Bühnentemperaments' und Reinigung der Bühnensprache " et "Abnehmen des Tons " (GBFA 23, 171-172, 169 et 174-175) qui traitent de la diction des acteurs - entre langue de scène et sociolectes populaires - pour exhausser l'importance du gestus linguistique dans son rapport avec le gestus fondamental et les gestus sociaux. 
Jedenfalls kann ein Theater, das alles aus dem Gestus nimmt, der Choreographie nicht entraten. Schon die Eleganz einer Bewegung und die Anmut einer Aufstellung verfremdet, und die pantomimische Erfindung hilft sehr der Fabel. (GBFA 23, 96).

L'image muette et fixe, débarrassée de la vocalité de dialogues, qui précisent d'une manière trop univoque la sémantisation plus diffuse des arrangements scéniques, peut être ainsi d'une salubre abstraction. C'est certainement, de par la focalisation sur la gestuelle, la mimique et les attitudes corporelles, le point fort des photos de scène ${ }^{42}$. Le statisme inhérent à l'image fixe peut avoir, surtout pour les imitations du Modèle, un effet de concentration sur les options proxémiques, avant qu'un mouvement, souvent arbitraire et parasitaire, ne les dissolve, comme Brecht l'écrit dans un court texte du Modèle-Courage:

Alle Gruppen zeigten auf unserem Theater eine starke Tendenz, sich aufzulösen, teils weil der Schauspieler sich von Gängen und Stellungsveränderungen eine Belebung des Zuschauerinteresses erwartet, teils weil er für sich sein und das Auge des Zuschauers mit sich wegziehen will. (GBFA 25, 184)

Les photos de scène combinent par ailleurs, avec des effets différents, les deux types d'images étudiés par G. Deleuze: elles sont surtout des "images-temps " mais elles sont aussi des "images-mouvement " de par les traces rémanentes qui sont visibles surtout dans la gestuelle, la position des corps voire celle des vêtements ainsi, avons-nous dit, que les positionnements proxémiques avec effet de filé. Si chaque photo n'en saisit qu'un moment isolé, l'observateur peut reconstituer intellectuellement le mouvement, comme s'il disposait de cette faculté physiologique qui a précédé puis déterminé la naissance du cinéma: la capacité de faire naître imaginairement le mouvement par la succession rapide d'images fixes destinées initialement à analyser celui-ci avec précision (Marey, Muybridge). C'est un aspect fondamental de la dialectique modèle / imitation qui augmente considérablement la latitude de la seconde à reconstituer spécifiquement ce que le premier a été contraint d'esquisser.

Néanmoins, la photo de scène ne génère pas ce que Deleuze décrit comme une "image pure [...], l'image entière et sans métaphore, qui fait surgir la chose en elle-même " en la débarrassant d'une foule de surdéterminations et "d'enchaînements sensori-moteurs " "3. Ce serait plutôt l'inverse: elle visualise pleinement le gestus en s'intégrant dans les " enchaînements sensori-moteurs " du montage d'une séquence qui surdétermine chacun de ses éléments. Partisane de séries rapprochées de photos, R. Berlau parle de " faire une série complète de photos d'un détail scénique " (Durchfotografieren eines Szenendetails) pour rendre

42. Je rejoins ici les réflexions de H.T. Lehmann qui souligne un certain primat du gestique sur le discours des dialogues pour produire des signifiés socio-politiques: "Den Gestus hat Brecht als zentrales Konzept seiner Theatertheorie nicht in Anschlag gebracht, um begrifflich Gewusstes mit dem Körper illustrierend darzustellen. Vielmehr weil eben das Wort, der Diskurs, die Theorie nicht ausreicht, um die entscheidenden politischen Probleme und Konflikte darzustellen, wird das Theaterspiel und also vor allem das Gestische benötigt. " (Hans Thies Lehmann, Brecht Lesen, Berlin, Theater der Zeit, p. 228).

43. Cf. Gilles Deleuze, L'Image-temps, Paris, Éd. de Minuit, 1985, p. 32-33. 
lisibles les détails du gestus de certaines scènes : « Detailaufnahmen werden nach der Überlegung gemacht, welche Geste oder welcher Ausdruck den Charakter der Figur zeigt, speziell die Widersprüche des Charakters. " (TA, 294-295).

Ceci explique l'insistance brechtienne à avoir à sa disposition un maximum de photos de scène. Il demanda ainsi à R. Berlau d'en réaliser plusieurs centaines de l'acteur de théâtre et de cinéma Charles Laughton pour le premier Galilée hollywoodien mis en scène par Joseph Losey (juin 1947). Celle-ci tourna également un film de 35 minutes qui montrait les principaux arrangements scéniques et était entrecoupé d'extraits du texte de la pièce. L'objectif était d'une part de permettre d'utiliser ces photographies comme documents de travail avant chaque répétition et d'autre part de s'assurer qu'une deuxième mise en scène (new-yorkaise) en décembre 1947 - toujours avec Laughton dans le rôle-titre mais en l'absence de Brecht cette fois - respecterait les options retenues à Hollywood.

C'est ce qui fait de la mise en scène américaine de Galilée, centrée sur le travail de Brecht et de R. Berlau avec Laughton, le prototype ou avant-modèle des Modellbücher. Il sera publié en 1956 au Henschel Verlag sous le titre " Aufbau einer Rolle. Laughtons Galilei ", au moment où Brecht livre une troisième et ultime version du texte de la pièce. Photos et film servirent en 1955-56 au Berliner Ensemble pour une reprise de la pièce avec Ernst Busch dans le rôle-titre, travail poursuivi, après la maladie et la mort de Brecht, par Erich Engel jusqu'à la première en $1957^{44}$. Brecht écrivit à son éditeur Bruno Henschel (17 mai 1956) :

Es war geplant, das Modellbuch zu ergänzen mit Beschreibungen und Fotos des Galilei von Ernst Busch. Das Buch würde zweifellos dadurch sehr viel wertvoller werden. Man wird sehen, wie zwei bedeutende Schauspieler dieselbe Rolle ganz individuell aufbauen. (GBFA 30, 452).

La comparaison par les photos entre l'avant-Modèle et le Modèle est éclairante : tandis que Laughton est omniprésent dans des photos de scène centrées sur son corps en mouvement plus que sur son visage, les photos du Modellbuch ultérieur semblent révéler un jeu moins extériorisé de l'acteur principal, peutêtre plus statique et plus austère mais aussi plus axé sur les positions des groupements. Comme si la captation photographique hollywoodienne connotait à l'insu de R. Berlau le star system américain, Brecht ayant notoirement laissé à Laughton la bride sur le cou et s'étant montré nettement plus directif avec

44. Le film se trouve au Brecht-Archiv (BBA-AVM 13.0049). Brecht propose, début 1953, à l'acteur et directeur de théâtre polonais Alexandre Bardini, désireux de monter Vie de Galilée à Varsovie, de lui faire parvenir " un grand nombre de documents iconographiques de la représentation avec Charles Laughton dont un film entier " (GBFA 30, 163).

Pour une description précise et de première main du travail théâtral brechtien lors des répétitions de la pièce (1955-56), voir: Käthe Rülicke, "Leben des Galilei. Bemerkungen zur Schlußszene ", Sinn und Form, 2. Sonderheft Bertolt Brecht, Berlin 1957, p. 269-321. La deuxième édition des Materialien zu Brechts Leben des Galilei (1963) propose des documents sur les mises en scène de la pièce entre 1947 et 1978: Brechts Leben des Galilei, éd. par W. Hecht, Frankfurt am Main, Suhrkamp, 1981. 
le Galilée reterritorialisé linguistiquement et idéologiquement dans l'espace du Schiffbauerdamm ${ }^{45}$.

Par ailleurs, les commentaires de ce premier Modellbuch avant la lettre montrent le rôle essentiel d'un acteur dans la construction d'un rôle, dans les modifications d'idées de mise en scène voire du texte de la pièce. Car le texte initial peut être " usé " par la mise en scène et les propositions des comédiens :

Was wir machten, war ein Text, die Aufführung war alles. [...]; in der Aufführung fand der Verschlei $\beta$ des Textes statt... (GBFA 25, 10- 12). ${ }^{46}$

En donnant la primauté à la représentation sur le texte de la pièce, l'avantModèle issu d'une mise en scène en langue anglaise fait également apparaître quelques évidences qui contribuent à défétichiser le texte original du Galilée. Un bon traducteur avait à " traduire des gestes ", dit Brecht, car la langue était " théâtrale, là où elle exprime la relation des locuteurs entre eux " (ibid., p. 12). En exhaussant le processus d'un travail théâtral essentiellement articulé sur le "contenu gestique " d'une pièce, Brecht revitalise d'un même mouvement sa théorie ${ }^{47}$.

La description de cet avant-Modèle du Galilée fait également apparaître un double manque: d'abord la réalisation d'un film et pas seulement de photos sur la mise en scène repose le problème fondamental des images animées ou fixes, parlantes ou muettes, déjà évoqué. La conscience d'un tel manque conduira le Berliner Ensemble, après l'échec des adaptations cinématographiques d'Alberto Cavalcanti et de Wolfgang Staudte à faire tourner en studio par les " Meisterschüler " Peter Palitzsch et Manfred Wekwerth - le film ne sera terminé qu'en 1961 - la mise en scène berlinoise de Mère Courage ${ }^{48}$. Mais l'avantModèle de Galilée pointe aussi indirectement le problème de la couleur, toutes les photographies de R. Berlau étant non seulement des images fixes mais aussi en noir et blanc. Or, " Aufbau einer Rolle " insiste sur le rôle essentiel du choix

45. Il serait intéressant de proposer une analyse comparative des variations dramaturgiques entre les deux versions scéniques d'une pièce aussi souvent analysée, mais à partir des photos et commentaires de "Aufbau einer Rolle " (Brecht/Berlau) et du Modellbuch non publié du Galilée (Eisler/H. Hill) (BBA - A1389), en particulier en ce qui concerne le jeu de Laughton et de Busch: "Busch ist ein norddeutscher Arbeiter, ein Puritaner, kein Genußmensch wie Laughton ", écrivit H. Eisler dans l'avant-propos (ibid., p. 11).

46. Brecht note également comment un acteur de l'envergure de Laughton pourra refuser, à la reprise new-yorkaise de la pièce, d'en laisser modifier radicalement le gestus fondamental en élaguant la version du texte retenue dans le premier travail théâtral hollywoodien : "Ihm war so wichtig, da $\beta$ die Bedrohung der Besitzverhältnisse durch die Lehre der Erdumdrehung demonstriert wurde, da $\beta$ er eine New Yorker Produktion, welche diese Szene streichen sollte, ablehnte. " (GBFA 25, 50).

47. La manière dont ce travail peut nourrir le travail proprement théorique est perceptible dans le paragraphe le plus fourni du Petit Organon (§ 63), qui décrit, en s’adressant exceptionnellement à un " tu " fictif, le " contenu gestique " de la scène initiale de Vie de Galilée.

48. Dans une lettre d'avril 1954 au département cinéma du Ministère de la Culture de RDA, Brecht semble désireux de compléter la documentation photographique du travail théâtral du Berliner Ensemble par un filmage plus ou moins systématique des représentations à usage interne: "Das könnte wertvolles Material für unsere Schauspieler ergeben. " (GBFA 30, 240). 
des couleurs ("Farbenschema ") pour les costumes et la scénographie: "Jede Szene mußte einen Grundton haben ", "Aber die Gesamtheit der Szenen mußte dann ihre Entwicklung haben in der Farbe ", " Der gesellschaftliche Aufstieg des Galilei wurde auch in den Farben sichtbar " $(G B F A 25,18)$.

Un troisième aspect du Galilée américain illustre une utilisation des Modèles " dans des conditions particulières " - des représentations en langue étrangère qui font de leur imitation un phénomène complexe dont la traduction n'est qu'un élément. Le meilleur exemple en est la mise en scène de Mère Courage en Hollande, supervisée par R. Berlau. Celle-ci écrivit ce qui est peut-être le compte-rendu le plus remarquable d'un travail théâtral délibérément calqué sur celui du Berliner Ensemble, mais avec des extensions pratiques qui réexaminent les positions du collectif berlinois sans les remettre en question ${ }^{49}$. Ces extensions furent dictées par les conditions particulières de la situation hollandaise : tradition naturaliste et aristotélicienne du jeu de l'acteur, manque de familiarité avec les exigences du théâtre épique brechtien et tendance à surjouer la dévastation $d u$ maternel et non le versant affairiste obstiné et mortifère de Mère Courage (voir supra $)^{50}$, méconnaissance du statut des accessoires pour signifier des éléments capitaux de gestus fondamental ou social, nécessité de retravailler la traduction en fonction de la capacité du nouveau texte à passer vocalement la rampe sans dénaturer le sens du texte allemand, par exemple en truffant le néerlandais de petits " mots de remplissage " censés expliciter ou contextualiser les dialogues. R. Berlau s'y avéra être non seulement la photographe de gestus scéniques pertinents mais aussi la fine connaisseuse de la langue brechtienne, insistant sur les problèmes extratextuels liés à sa traduction et le rôle concret de ceux qui la mettent en voix sur scène:

Das ist die Knappheit der Sprache. Nach kurzer Zeit fanden die Schauspieler, dass man die kleinen Zwischenwörter streichen konnte. Für einen Brecht-Übersetzer liegt die Schwierigkeit darin, da $\beta$ Knappheit und Poesie sich die Hand geben müssen. Die niedrige, grobe und die schöne, große Sprache stimmen überein mit dem Vorgang. Oft läuft das Sprachliche dem Übersetzer sozusagen vom Inhalt weg und verliert das Gestische, was den Schauspieler sofort hilflos macht. (TA, 331)

Le comédien est donc un facteur déterminant dans l'imitation/transmission $d u$ Modèle. Il est le garant corporel que la langue ne défigure pas le gestus qui doit habiter la vocalité, très travaillée au Berliner Ensemble ${ }^{51}$, autant que la gestualité. Comme l'avait exemplairement montré le travail californien avec

49. Cf. " Benutzung von Modellen unter besonderen Bedingungen ", in TA, 328-332.

50. Ce qui a poussé le collectif de mise en scène à insérer une nouvelle réplique de fin - "Ich muß wieder in'n Handel kommen " - qui fut intégrée au Modellbuch (TA, 329). Pour une mise en scène de la pièce à Göttingen, en septembre 1956, Brecht pointera des raisons contextuelles de respecter cet élément essentiel du gestus fondamental: "Es wird jetzt, wo das deutsche Wirtschaftswunder und die Politik der Stärke in so drohender Weise Arm in Arm auftreten, besonders wichig, die Courage als Händlerin zu spielen. " (GBFA 24, 274).

51. Dès le début (déc. 1949), le Berliner Ensemble engagea spécialement un professeur de diction ("Sprechbildnerin "), Helga Gernerich, pour former et éduquer la voix des comédiens. Voir l'article de celle-ci, " Stimmbildung und Sprecherziehung ", in TA, 390-393. 
Charles Laughton sur Galilée, les «meilleures traductions sont celles qui sont nées au théâtre " $(T A, 332)$. Le jeu de l'acteur, qui a assimilé tous les gestus de la pièce, est parfois plus " fidèle " au Modèle que le texte-cible du traducteur. Les métaphores filées de R. Berlau sont sans équivoque:

Alle Übersetzungen von Theaterstücken mu $\beta$ man kämmen, bürsten und mu $\beta$ sie reinigen von überflüssigen Wörtern und erklärenden Umschreibungen. Eine Geste macht den Satz klar. ${ }^{52}$

En conclusion, on pourrait retenir que la confection et diffusion des Modellbücher et du Theaterarbeit devenait, dans sa concrétion évolutive et interactive, un concentré réflexif d'une pratique théâtrale disséminée implicitement dans plusieurs collectifs avec lesquels l'auteur/metteur en scène testait la circulation de ses idées scéniques et surtout la possibilité de propager un vrai penser théâtral progressiste. Que celuici dût être largement polycéphale, la formule brechtienne qu'aimait à citer W. Benjamin en donne la plus parfaite illustration: « Er dachte in andern Köpfen, und auch in seinem Kopf dachten andere. Das ist das richtige Denken. " (GBFA 21, 420).

À l'instar du changement de paradigme intervenu dans sa définition du socialisme, on pourrait ainsi voir dans les Modèles de Brecht non pas l'emblème d'un "grand ordre " à imposer, autant théâtral que social, mais plutôt celui d'une "grande production " (GBFA 26, 468) qui ne pouvait être que collective. Montrer le théâtre en travail permanent et polyvalent impliquait une dialectique opiniâtre entre Modèles et imitations qui faisait de celui-ci foncièrement un lieu de circulation des imitations comme retravail infini. En ce sens, un tel travail pouvait bien figurer ce que E. Bloch disait en 1938 du théâtre brechtien: " es sind TheoriePraxis-Manöver auf der Bühne ${ }^{53}$ ». Il avait à intégrer toute déviance qui ne remette pas en cause les fondamentaux du thêâtre brechtien mais sans ériger ceux-ci en totalité doctrinale ou en "grand ordre " théâtral. Là où une imitation trop fidèle témoignerait à son insu de la méconnaissance du Modèle, Brecht invitait à produire une vraie connaissance en s'ouvrant à la productivité intrinsèque de toute forme de mise à distance, même si celle-ci pouvait prendre les apparences d'une certaine " asocialité " artistique, car celle-ci ne se démarquait souvent du reproduire que pour intensifier le produire. Ce fut, on le sait, l'un des grands thèmes de son écriture théâtrale et de sa pensée ${ }^{54}$. L'imitation de Modèles n'a pas à simuler le respect répétitif d'une intangibilité du sens d'une pièce et de sa mise en signes scéniques mais à en explorer toutes les potentialités, fût-ce "gegen den Strich ", aurait dit Benjamin. Elle doit être une véritable "pensée intervenante " sur toutes les composantes d'un Modèle, sans a priori mais en pleine conscience des présupposés de leur agencement.

52. TA 332.

53. Ernst Bloch, "Ein Leninist der Schaubühne ", in Erbschaft dieser Zeit, Frankfurt am M., Suhrkamp, p. 254.

54. Cf. la note de journal du 11.6.49 - en plein travail sur Mère Courage - sur la productivité des asociaux: "Der Asoziale erfreut ebenfalls; es wird als genügend angesehen, da $\beta$ er sich produziert. " (GBFA 27, 305). Cf. aussi le § 25 du Petit Organon: "Selbst aus dem Asozialen kann die Gesellschaft so Genu $\beta$ ziehen, wofern es vital und mit Größe auftritt. " (GBFA 23, 71). 